\title{
MHD Instabilities in Tokamak Plasmas with Hollow Current Density Profiles
}

\author{
Goshi YAMADA, Yuji NAKAMURA ${ }^{1)}$ and Katsumi KONDO \\ ${ }^{1)}$ Graduate School of Energy Science, Kyoto University, Uji, Kyoto 611-0011, Japan
}

(Received 26 November 2012 / Accepted 13 June 2013)

\begin{abstract}
We analyze linear instability in low beta tokamak plasmas with nonmonotonic $q$-profiles using resistive magnetohydrodynamic equations. We consider $n=2$ (where $n$ is the toroidal mode number) modes for configurations where $q_{\min }$ is close to 1.5 and sufficiently below 2 so that the separation of the rational surfaces with $q=2$ is large. Similar instability characteristics arise when $q_{\min }$ is slightly and moderately above 1.5.

(c) 2013 The Japan Society of Plasma Science and Nuclear Fusion Research
\end{abstract}

Keywords: tokamak, low beta plasma, nonmonotonic safety factor profile, resistive MHD, linear instability

DOI: $10.1585 /$ pfr.8.1403156

\section{Introduction}

If the toroidal current density profile of a plasma is hollow, the safety factor $q$ profile is nonmonotonic, and the magnetic shear is negative in the central region. The formation of transport barriers has been confirmed in regions of negative magnetic shear in many tokamak experiments. However, the evolution of this type of configuration may lead to instabilities because multiple resonant surfaces with the same $q$ value exist in the plasma. In particular, double tearing modes may affect the confinement since they may cause relaxations of the electron temperature. Such relaxations have been observed in several experiments, where they have been attributed to double tearing modes [1,2].

The linear stability of double tearing modes has been analyzed using a simplified slab plasma model [3]. The distance $\Delta r_{s}$ between two rational surfaces with the same $q$ value is a significant quantity for double tearing modes. When $\Delta r_{s}$ is sufficiently large, the structure of the mode resembles that of individual single tearing modes localized on each resonant surface. When $\Delta r_{s}$ is sufficiently small, the interaction between two rational surfaces becomes strong, and a mode with much higher growth rate arises.

The linear growth rate and mode structure of the double tearing mode had not been systematically studied until approximately ten years ago. Since then, researchers have numerically investigated the linear behaviors of the double tearing mode for zero beta plasmas with nonmonotonic $q$ profiles in cylindrical geometry using the reduced magnetohydrodynamic equations $[4,5]$. However, to properly understand the nature of instabilities, plasmas must be investigated in general toroidal geometry. To this end, we apply toroidal geometry to low beta plasmas with nonmonotonic $q$-profiles and numerically solve incompressible resistive magnetohydrodynamic (MHD) equations.

author'se-mail: xa52427@pg7.so-net.ne.jp

\section{Model}

In this study, tokamak plasmas are analyzed using the linearized resistive MHD equations. The perturbed quantities $\tilde{\boldsymbol{v}}, \tilde{\boldsymbol{B}}$, and $\tilde{p}$, corresponding to the fluid velocity, the magnetic field, and the pressure, respectively, are obtained from

$$
\begin{aligned}
& \lambda \rho_{\mathrm{m}, \mathrm{eq}} \tilde{\boldsymbol{v}}=\frac{1}{\mu_{0}}\left(\nabla \times \boldsymbol{B}_{\mathrm{eq}}\right) \times \tilde{\boldsymbol{B}}+\frac{1}{\mu_{0}}(\nabla \times \tilde{\boldsymbol{B}}) \times \boldsymbol{B}_{\mathrm{eq}}-\nabla \tilde{P}, \\
& \lambda \tilde{\boldsymbol{B}}=\nabla \times\left(\tilde{\boldsymbol{v}} \times \boldsymbol{B}_{\mathrm{eq}}\right)-\frac{\eta}{\mu_{0}} \nabla \times \nabla \times \tilde{\boldsymbol{B}}, \\
& \nabla \cdot \tilde{\boldsymbol{v}}=0,
\end{aligned}
$$

where $\lambda$ is the growth rate to be determined. Also, $\eta$ is the resistivity, and the equilibrium quantities $\rho_{\mathrm{m}, \mathrm{eq}}$ and $\boldsymbol{B}_{\text {eq }}$ denote the mass density and magnetic field, respectively. The resistivity and equilibrium mass density are assumed constant. The equilibrium magnetic field possesses up-down symmetry and satisfies

$$
\begin{aligned}
& \frac{1}{\mu_{0}}\left(\nabla \times \boldsymbol{B}_{\text {eq }}\right) \times \boldsymbol{B}_{\text {eq }}=\nabla p_{\text {eq }}, \\
& \nabla \cdot \boldsymbol{B}_{\text {eq }}=0,
\end{aligned}
$$

where $p_{\text {eq }}$ is the equilibrium pressure.

The flux coordinate system $(\rho, \theta, \zeta)$ is employed for the geometry considered here. Here $\rho$, which is the averaged minor radius normalized by the edge value, labels the magnetic flux surface, i.e., the surface of constant pressure, and $\theta$ and $\zeta$, which range from $-\pi$ to $\pi$, are poloidal and toroidal angle-like coordinates, respectively. Because of the up-down symmetry of the equilibrium, the perturbed quantities can be expanded as the following Fourier series:

$$
\begin{aligned}
& \tilde{v}^{\rho}=\sum_{m} v_{m}^{\rho}(\rho) \cos \left(m \theta-n_{0} \zeta\right), \\
& \tilde{v}^{\theta}=\sum_{m} v_{m}^{\theta}(\rho) \sin \left(m \theta-n_{0} \zeta\right),
\end{aligned}
$$




$$
\begin{aligned}
\tilde{v}^{\zeta} & =\sum_{m} v_{m}^{\zeta}(\rho) \sin \left(m \theta-n_{0} \zeta\right), \\
\tilde{B}^{\rho} & =\sum_{m} B_{m}^{\rho}(\rho) \sin \left(m \theta-n_{0} \zeta\right), \\
\tilde{B}^{\theta} & =\sum_{m} B_{m}^{\theta}(\rho) \cos \left(m \theta-n_{0} \zeta\right), \\
\tilde{B}^{\zeta} & =\sum_{m} B_{m}^{\zeta}(\rho) \cos \left(m \theta-n_{0} \zeta\right), \\
\tilde{p} & =\sum_{m} p_{m}(\rho) \cos \left(m \theta-n_{0} \zeta\right),
\end{aligned}
$$

where $m$ and $n_{0}$ are the poloidal and toroidal mode numbers, respectively, and

$$
\begin{aligned}
& \tilde{v}^{\rho}=\tilde{\boldsymbol{v}} \cdot \nabla \rho, \quad \tilde{v}^{\theta}=\tilde{\boldsymbol{v}} \cdot \nabla \theta, \quad \tilde{v}^{\zeta}=\tilde{\boldsymbol{v}} \cdot \nabla \zeta, \\
& \tilde{B}^{\rho}=\tilde{\boldsymbol{B}} \cdot \nabla \rho, \quad \tilde{B}^{\theta}=\tilde{\boldsymbol{B}} \cdot \nabla \theta, \quad \tilde{B}^{\zeta}=\tilde{\boldsymbol{B}} \cdot \nabla \zeta .
\end{aligned}
$$

The plasma is assumed to be surrounded by a perfectly conducting wall. Thus, at the wall, we have

$$
v_{m}^{\rho}(1)=0, \quad B_{m}^{\rho}(1)=0 .
$$

The boundary conditions at the magnetic axis $\rho=0$ are given by

$$
\begin{array}{ll}
v_{m}^{\rho}=\rho v^{\theta}{ }_{m}=B^{\rho}{ }_{m}=\rho B^{\theta}{ }_{m}=0, & |m| \neq 1, \\
\frac{\mathrm{d} v_{m}^{\rho}}{\mathrm{d} \rho}=\frac{\mathrm{d}\left(\rho v_{m}^{\theta}\right)}{\mathrm{d} \rho}=\frac{\mathrm{d} B_{m}^{\rho}}{\mathrm{d} \rho}=\frac{\mathrm{d}\left(\rho B^{\theta}{ }_{m}\right)}{\mathrm{d} \rho}=0, & |m|=1, \\
v_{m}^{\zeta}=B_{m}^{\zeta}=p_{m}=0, & m \neq 0, \\
\frac{\mathrm{d} v_{m}^{\zeta}}{\mathrm{d} \rho}=\frac{\mathrm{d} B_{m}^{\zeta}}{\mathrm{d} \rho}=\frac{\mathrm{d} p_{m}}{\mathrm{~d} \rho}=0, & m=0 .
\end{array}
$$

The above conditions are derived by requiring that the physical quantities be well-behaved near the magnetic axis. The growth rate and Fourier components of the perturbed quantities are determined by the inverse power method [6]. Although this method allows us to extract unstable modes other than the most unstable one, in this study, we consider only the fastest growing mode.

\section{Numerical Results}

Plasma equilibria are constructed by the variational moments equilibrium code (VMEC) [7]. Threedimensional equilibria are obtained by minimizing the potential energy of the plasma using the steepest descent method. The cross section of the plasma is circular with a radius of $0.75 \mathrm{~m}$. The major radius at the plasma center is $R_{0}=2.5 \mathrm{~m}$. The constant mass density is set to $\rho_{\mathrm{m}, 0}=10^{-6} \mathrm{~kg} / \mathrm{m}^{3}$. The magnetic field strength at the magnetic axis is $B_{0}=4 \mathrm{~T}$. The pressure profile (normalized by central pressure) is shown in Fig. 1. This profile displays a large slope in the middle region. The norm of $\nabla \rho$ is almost uniform throughout the plasma. This implies the pressure gradient is large in the middle region. Figure 2 shows the safety factor profiles used in the analysis.

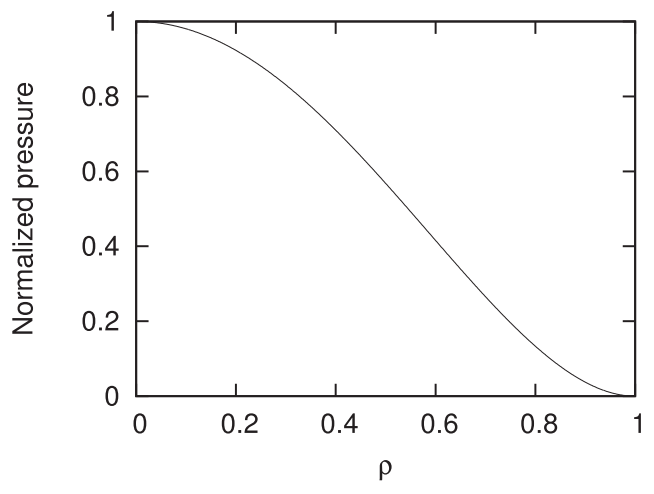

Fig. 1 Pressure profile normalized by pressure at the magnetic axis.

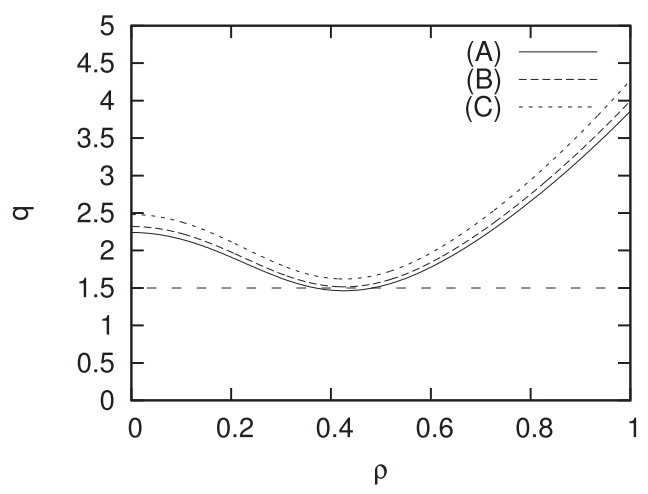

Fig. 2 Safety factor profiles.

The minimum value of safety factor $q_{\min }$ is slightly below and slightly above 1.5 for Cases (A) and (B), respectively. For Case (C), $q_{\min }$ is moderately above 1.5 . This study is restricted to modes with toroidal mode number $n_{0}=2$.

In Fig. 3 the growth rate is plotted as a function of magnetic Reynolds number $S=\tau_{R} / \tau_{H}$ for various values of $\beta_{0}$ for Case (A). Here, $\tau_{H}=\sqrt{\mu_{0} \rho_{\mathrm{m}, 0} / B_{0}^{2}} R_{0}$ and $\tau_{R}=\mu_{0} R_{0}^{2} / \eta$ are the Alfvén time and resistive diffusion time, respectively. Also, $\beta_{0}$ is the beta value at the magnetic axis. Figure 4 shows the mode structure of the radial component of the perturbed fluid velocity for $\beta_{0}=0.62 \%$ and $S=3 \times 10^{6}$. The mode structures for the other cases are similar to this one. The $m=3$ component is dominant and this component has a large value between the resonant surfaces where $q=1.5$. This structure is close to the mode structure of displacement in slab geometry when the distance between rational surfaces is small [3]. Figure 5 shows the dependence of the growth rates on $S$ for Case (C). In contrast to the situation in Case (A), the growth rate decreases with $\beta_{0}$ remarkably. Figure 6 shows the mode structures of $\tilde{v}^{\rho}$ for $S=3 \times 10^{6}$ at three values of $\beta_{0}$. The $m=3$ component is large at $\beta_{0}=3.13 \%$. This component is negligibly small at $\beta_{0}=0.62 \%$. The $m=4$ component is localized at the resonant surfaces where $q=2$ and has two nodes in the vicinities of the resonant surfaces 


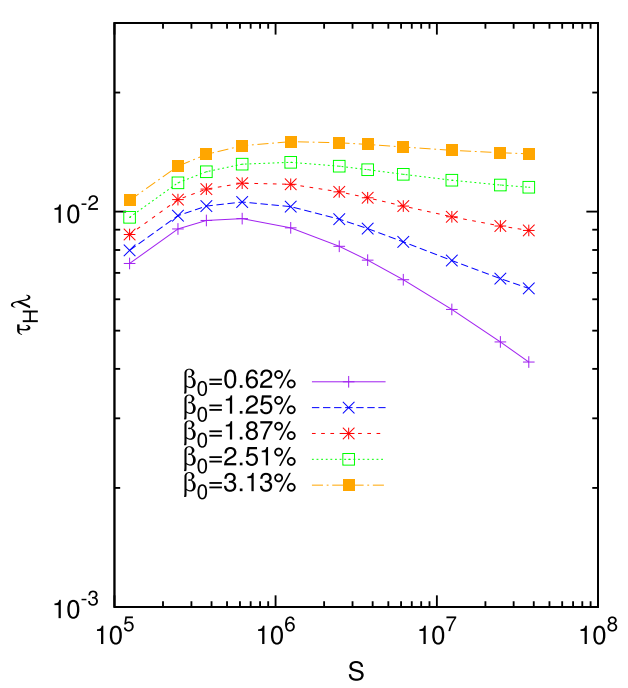

Fig. $3 S$ dependence of the growth rates for various values of $\beta_{0}$ for Case (A).

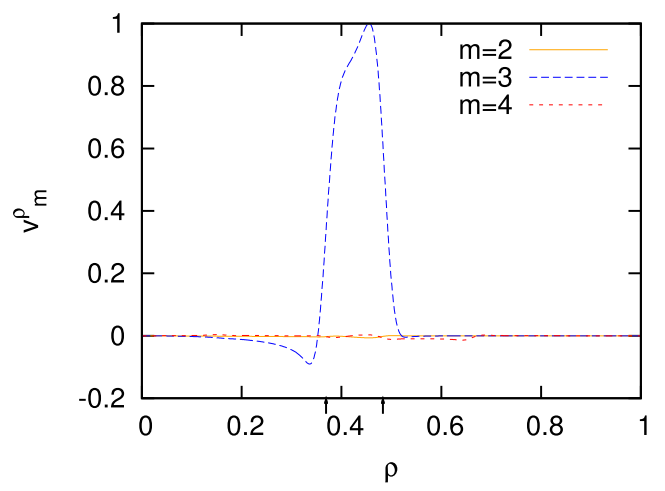

Fig. 4 Mode structure of $\tilde{v}^{\rho}$ for Case (A) and $S=3 \times 10^{6}$ at $\beta_{0}=$ $0.62 \%$. Arrows indicate the locations of the resonant surfaces with $q=1.5$.

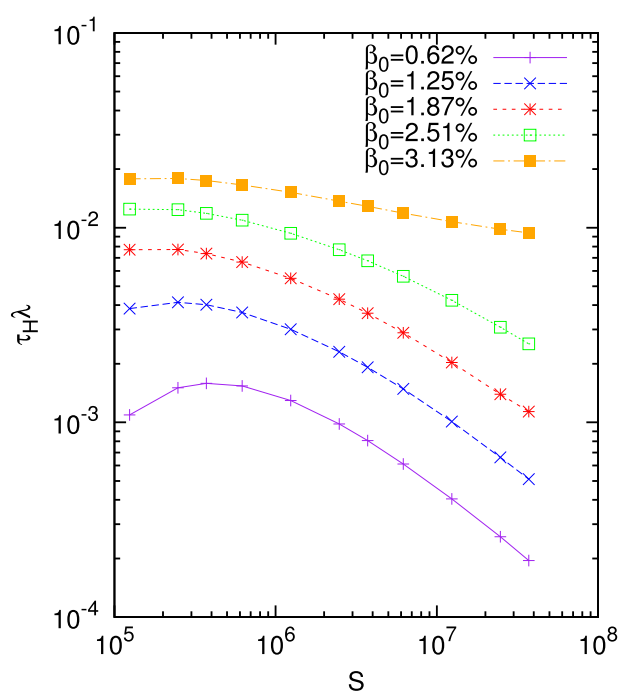

Fig. $5 S$ dependence of the growth rates for various values of $\beta_{0}$ for Case (C). (a)

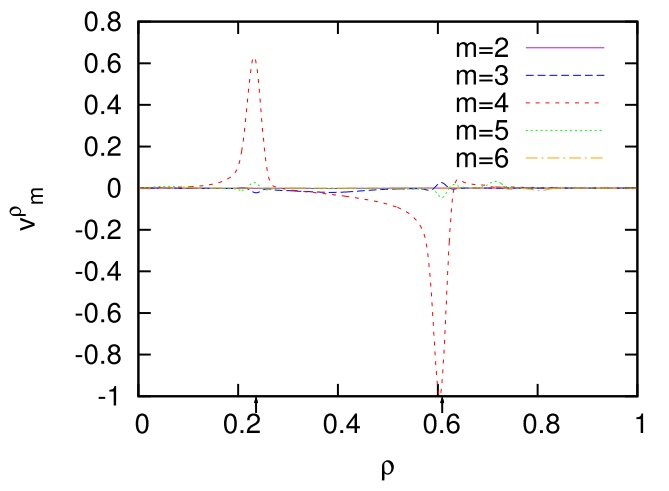

(b)

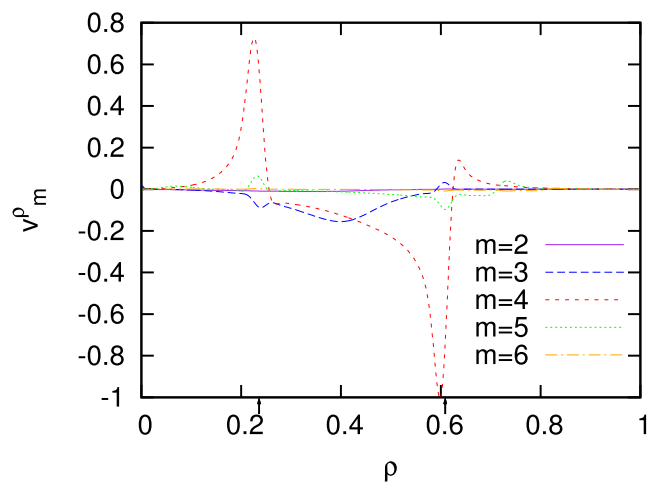

(c)

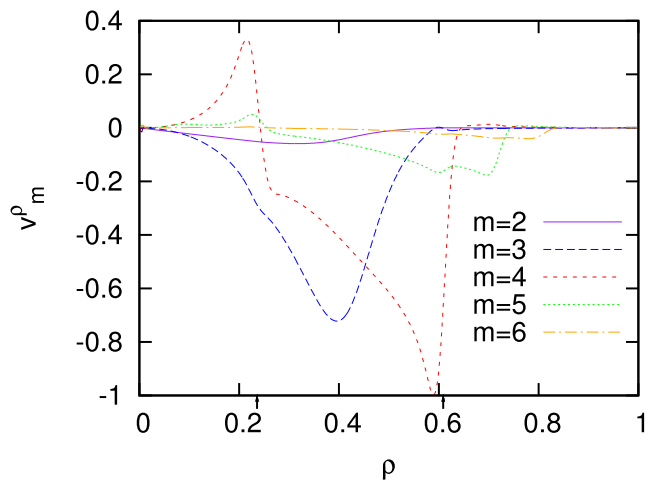

Fig. 6 Mode structures of $\tilde{v}^{\rho}$ for Case (C) and $S=3 \times 10^{6}$ at (a) $\beta_{0}=0.62 \%$, (b) $\beta_{0}=1.87 \%$, and (c) $\beta_{0}=3.13 \%$. Arrows indicate the locations of the resonant surfaces where $q=2$.

at $\beta_{0}=0.62$ and $1.87 \%$. This structure is close to the mode structure of displacement in slab geometry when the distance between rational surfaces is sufficiently large [3]. When the region where $q$ is close to some rational number is broad, in general, the plasma becomes more unstable. Therefore, the configurations where $q_{\min }$ is very close to 1.5 are expected to be more unstable. Next we consider Case (B) corresponding to $q_{\text {min }} \simeq 1.5$. Figure 7 shows the dependence of growth rates on $S$ for Case (B). The growth rate varies with $\beta_{0}$ remarkably as in Case (C). Figure 8 


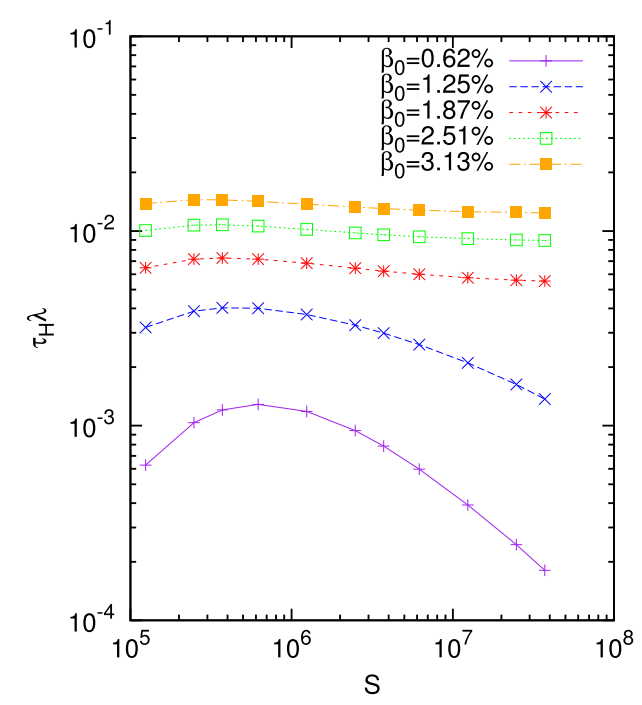

Fig. $7 S$ dependence of the growth rates for various values of $\beta_{0}$ for Case (B).

shows the mode structures of $\tilde{v}^{\rho}$ for $S=3 \times 10^{6}$ at three values of $\beta_{0}$. The $m=3$ component is dominant for the cases $\beta_{0}=1.87$ and $3.13 \%$. This component is small compared with the $m=4$ component, which is localized at the resonant surfaces where $q=2$, at $\beta_{0}=0.62 \%$. The $m=3$ component becomes smaller as $\beta_{0}$ decreases for Cases (B) and (C), and this component is especially small in configurations in which the growth rate is highly sensitive to $S$. The growth rate decreases as $S$ is decreased in low $S$ at small $\beta_{0}$ for all the cases. We suppose this is because the second term on the right-hand side of Eq. (2) has a stabilizing effect as a dissipation term when the resistivity is sufficiently large. There is spiky behavior in the vicinity of $\rho=0$ in some of the figures showing the mode structures. This kind of spiky behavior appears randomly independently of the configuration. We conjecture that it has nothing to do with the physics of plasma and it is some numerical noise.

The growth rates of ideal modes are plotted as a function of $q_{\min }$ for $\beta_{0}=1.87$ and $3.13 \%$ in Fig. 9. The growth rates in the left part of this figure are larger than those in the right part for $\beta_{0}=3.13 \%$. The plasma is unstable in the range $1.5<q_{\min }<1.53$ and stable in the range $1.53<q_{\min }<1.62$ for $\beta_{0}=1.87 \%$. These facts are consistent with the prediction mentioned above. The growth rates are plotted as a function of $q_{\min }$ for $S=3 \times 10^{6}$ and $\beta_{0}=0.62 \%$ in Fig. 10. For the region $q_{\min }>1.5$ the growth rate abruptly increases in the neighborhood of 1.5. Apart from this behavior around 1.5, the growth rates in the left and right parts above $q_{\min }=1.5$ are not so different, unlike the above cases of ideal modes. The growth rate abruptly changes in the region $1.47<q_{\min }<1.5$. The mode structure of $\tilde{v}^{\rho}$ also abruptly changes in this region. The mode structures in the range $1.41<q_{\min }<1.47$ are similar to the profile in Fig. 4. The mode structures (a)

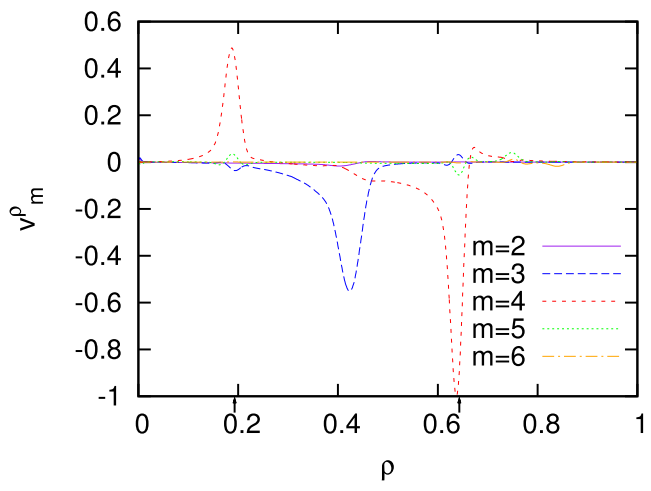

(b)

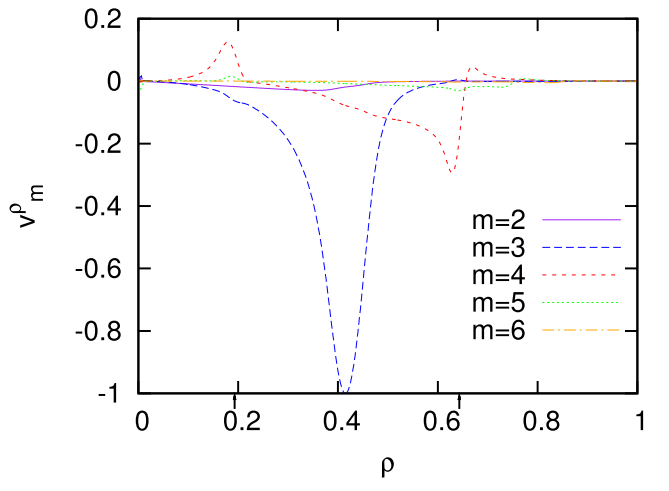

(c)

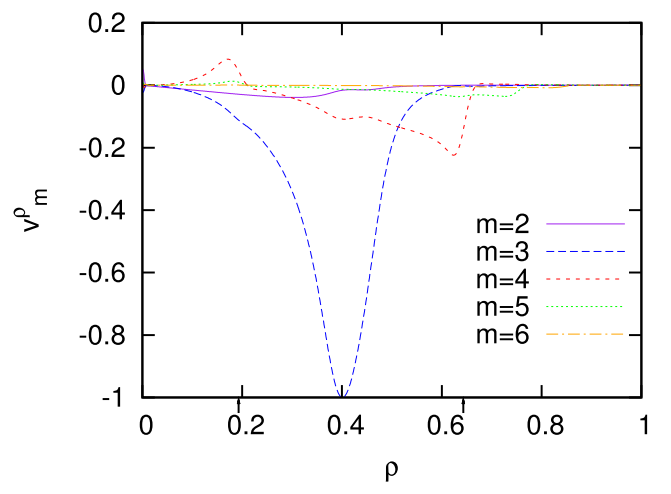

Fig. 8 Mode structures of $\tilde{v}^{\rho}$ for Case (B) and $S=3 \times 10^{6}$ at (a) $\beta_{0}=0.62 \%$, (b) $\beta_{0}=1.87 \%$, and (c) $\beta_{0}=3.13 \%$. Arrows indicate the locations of the resonant surfaces where $q=2$.

in the range $1.51<q_{\min }<1.62$ resemble the profiles in Figs. 6 (a) and 8 (a). The $m=3$ component becomes small as $q_{\text {min }}$ becomes large.

\section{Summary}

The linear instability characteristics of low beta tokamak plasmas with hollow current density profiles were numerically investigated using resistive MHD equations. We examined modes with $n_{0}=2$ for configurations where $q_{\text {min }}$ 
is close to 1.5 . When $q_{\min }$ is slightly below 1.5 , the distance between the rational surfaces with $q=1.5$ is small and the $m=3$ component, which is a dominant component, has a large value between the rational surfaces. The growth rate does not vary with $\beta_{0}$ so much in this case. When $q_{\min }$ is above 1.5 and sufficiently below 2 so that the distance between the rational surfaces with $q=2$ is large, the $m=3$ and $m=4$ components are dominant. The $m=3$ component becomes smaller as $\beta_{0}$ decreases and the growth rate varies with $\beta_{0}$ remarkably. This trend emerges when $q_{\text {min }}$ is moderately above 1.5 and also when $q_{\min }$ is almost equal to 1.5 .

For the case of $q_{\min }>1.5$, the ideal modes tend to become more unstable when $q_{\min }$ is very close to 1.5 because the region where $q$ is almost equal to 1.5 is broad, whereas there is only a little such tendency for the resistive modes at very low beta. The instability properties of the resistive modes at very low beta suddenly change in the neighborhood of $q_{\text {min }}=1.5$, where the transition from modes with large growth rates to modes with much smaller growth rates occurs.

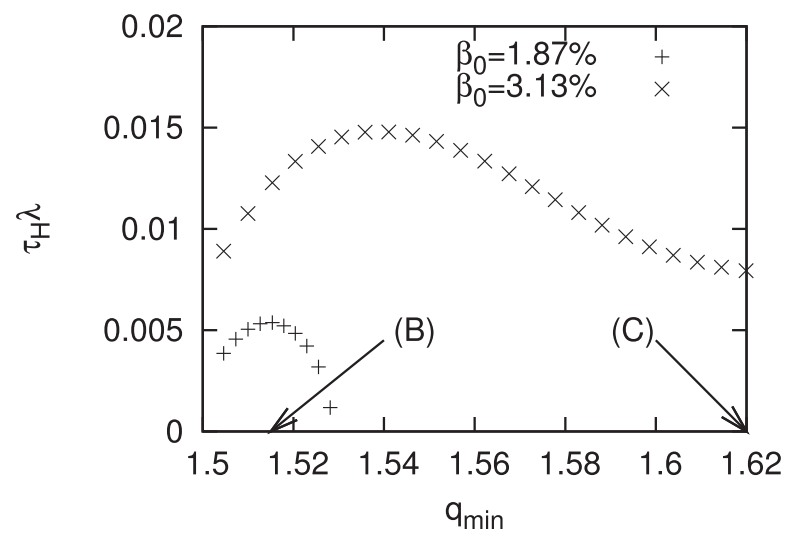

Fig. $9 q_{\min }$ dependence of the growth rates for ideal modes.

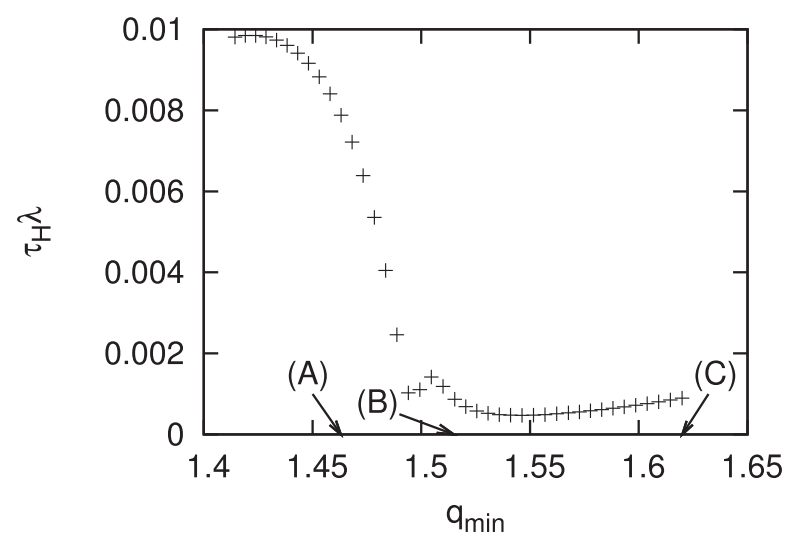

Fig. $10 q_{\text {min }}$ dependence of the growth rate for $S=3 \times 10^{6}$ and $\beta_{0}=0.62 \%$.

\section{Appendix}

We verify our numerical code by comparing the results of our code with those of another code. Numerical simulations based on the reduced resistive MHD in cylindrical geometry have been previously conducted [5]. Here we demonstrate that the numerical results obtained by our code resemble those in Ref. 5 for similar configurations.

We use a zero beta plasma with a circular cross section. The major radius at the plasma center is $R_{0}=2.5 \mathrm{~m}$ and the aspect ratio of the plasma is 10 . The constant mass density is set to $\rho_{\mathrm{m}, 0}=10^{-6} \mathrm{~kg} / \mathrm{m}^{3}$. The magnetic field strength at the magnetic axis is $B_{0}=4 \mathrm{~T}$. Figure A1 shows the safety factor profile. This profile is analogous to the $q$ profile of Case (IIIa) in Ref. 5. We consider modes with $n_{0}=1$.

In Fig. A2, the growth rates $\lambda_{1}$ and $\lambda_{2}$ of the modes with the largest growth rate and the second largest one, respectively, are plotted as a function of the magnetic Reynolds number, defined as $S=\tau_{R} / \tau_{H}$ with $\tau_{H}=$ $\sqrt{\mu_{0} \rho_{\mathrm{m}, 0} / B_{0}^{2}} R_{0}$ and $\tau_{R}=\mu_{0} R_{0}^{2} / \eta$. The $S$ dependence in Fig. A2 is qualitatively similar to the left portion of Fig. 15 (a) in Ref. 5. The orders of magnitude of $S$ and

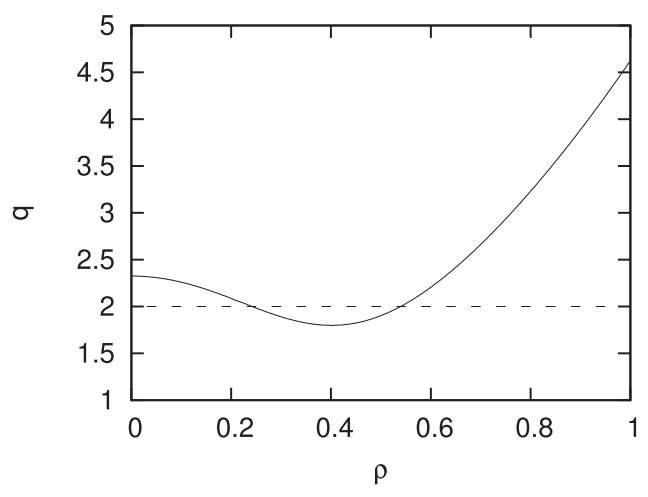

Fig. A1 Safety factor profile.

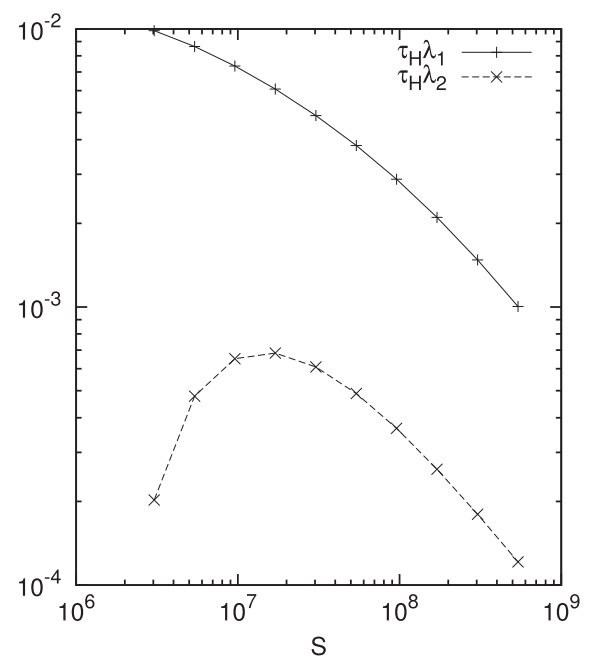

Fig. A2 $S$ dependence of the growth rates. 
(a)
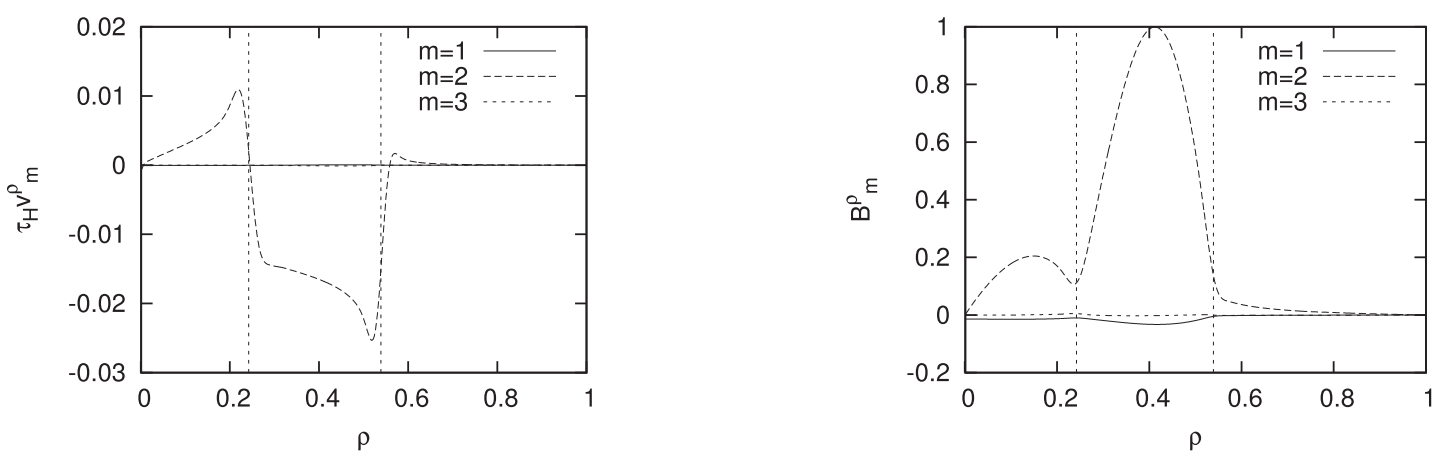

(b)
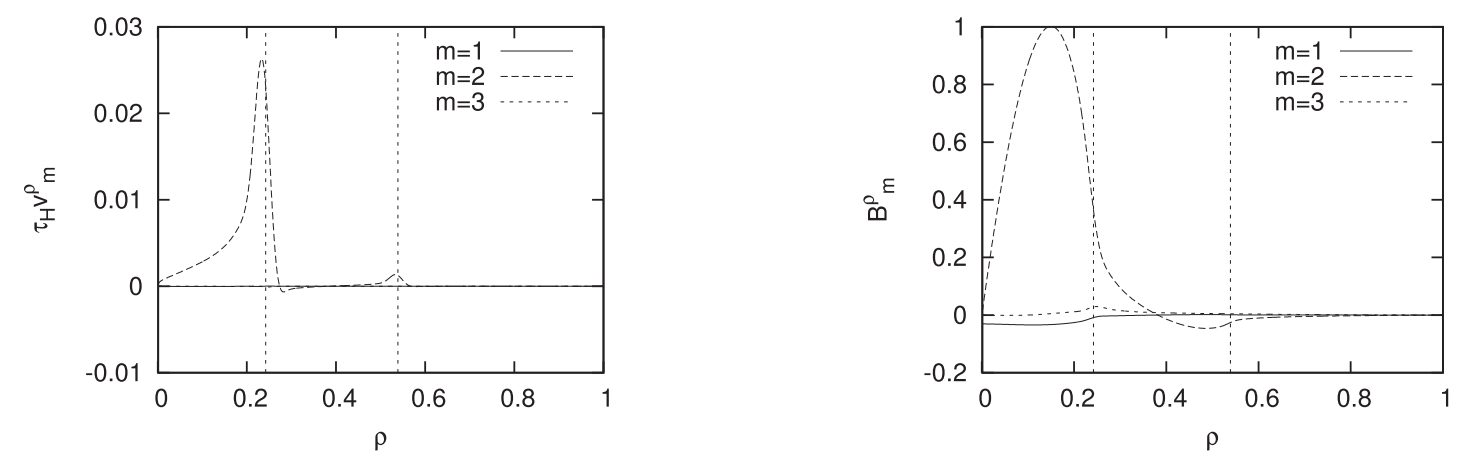

Fig. A3 Mode structures of $\tilde{v}^{\rho}$ and $\tilde{B}^{\rho}$ at $S=10^{8}$. The mode structures of the eigenmodes which grow at the largest growth rate and the second largest one are shown in (a) and (b), respectively. Vertical dotted lines indicate the locations of the resonant surfaces where $q=2$.

$S_{\mathrm{Hp}}$ in these figures differ because the magnetic Reynolds number is defined differently in the two studies. Figure A3 shows the mode structures of $\tilde{v}^{\rho}$ and $\tilde{B}^{\rho}$ obtained at $S=10^{8}$, which is almost equivalent to $S_{\mathrm{Hp}}$ at which the profiles in Figs. 4 (a) and 4 (c) in Ref. 5 are obtained. The $m=2$ components are dominant for both of the eigenmodes with the largest growth rate and the second largest one. The functions $\phi / r$ and $\psi / r$ for Case (IIIa) correspond to the Fourier components $v_{m}^{\rho}$ and $B_{m}^{\rho}$ with $m=2$. The profiles in Figs. 4 (a) and 4 (c) resemble their corresponding profiles in Fig. A3.
[1] Z. Chang et al., Phys. Rev. Lett. 77, 3553 (1996).

[2] M.R. de Baar, G.M.D. Hogeweij, N.J. Lopes Cardozo, A.A.M. Oomens and F.C. Schuller, Phys. Rev. Lett. 78, 4573 (1997).

[3] P.L. Pritchett, Y.C. Lee and J.F. Drake, Phys. Fluids 23, 1368 (1980).

[4] Y. Ishii, M. Azumi, G. Kurita and T. Tuda, Phys. Plasmas 7, 4477 (2000).

[5] A. Bierwage, S. Benkadda, S. Hamaguchi and M. Wakatani, Phys. Plasmas 12, 082504 (2005).

[6] L.A. Charlton, J.A. Holmes, H.R. Hicks, V.E. Lynch and B.A. Carreras, J. Comput. Phys. 63, 107 (1986).

[7] S.P. Hirshman and J.C. Whitson, Phys. Fluids 26, 3553 (1983). 